\title{
Medium-Energy Nuclear Data Library (MENDLIB): Phase I
}

\author{
E. R. Siciliano \\ E. D. Arthur
}

\section{DISCLAIMER}

\begin{abstract}
This report was prepared as an account of work sponsored by an agency of the United States Government. Neither the United States Government nor any agency thereof, nor any of their employees. makes any warranty, express or implied, or assumes any iegal liability or responsibility for the accuracy, completeness, or usefulness of any information, apparatus, product, or process disclosed, or represents that its use would not infringe privately owned rights. Reference trerein to any specific commercial product, process, or service by trade name, trademark. manufacturer, or otherwise does not necessarily constitute or imply its endorsement, recommendation, or favoring by the United States Government or any agency thereof. The views and opinions of authors expressed herein do not necessarily state or reflect those of the United States Government or any agency thereof
\end{abstract}
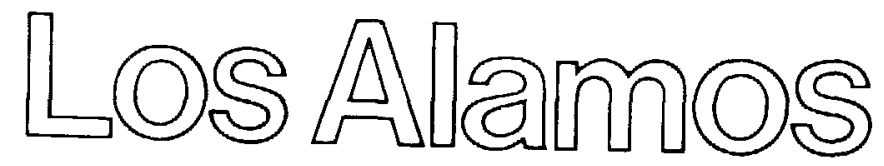


\title{
MEDIUM-ENERGY NUCLEAR DATA LIBRARY \\ (MENDLIB): PHASE I
}

by

E.R. Siciliano and E.D. Arthur

\begin{abstract}
This document describes an initial step towards the formation of a computerized on-line data library, which would contain published medium-energy experimental data, and which would serve the basic and applied needs of the medium-energy nuclear physics community. The data emphasized in this project will be from measured charged-particle and meson induced nuclear scattering and reactions; an area for which no such data base presently exists. Access to the data will be through a menudriven program in a user-friendly environment. The project is divided into three phases: Phase I involves compilation of Clinton P. Anderson Meson Physics Facility (LAMPF) data from niscleon and pion induced reactions, Phase II includes nucleon and pion data from other medium-energy facilities, and Phase III includes electron, light-ion, and possibly kaon and anti-nucleon data. The initial goals, the manner in which they would be pursued, and the resources needed to implement Phase I (the pilot phase) are discussed in detail. Possible expansion of Phase I to attain the envisioned goals of Phase II and III are briefly outlined. During all stages of the project, aiput from the community will be sought via the various facility user groups and the American Physical Society Division of Nuclear Physics. It is proposed that the Applied Nuclear Science Group (T-2) of the Los Alamos National Laboratory oversees the development ard implementation of this project, and the LAMPF VAX compriers be used as the host computers for on-line access.
\end{abstract}




\section{Motivation}

Over the past decade, there has been a wealth of experimental data produced as a result of the full scale operation of the so-called medium-energy accelerators, such as the Indiana University Cyclotron Facility (IUCF), the Institute for Nuclear Physics Accelerator at Jülich (Jülich), LAMPF, the Massachusetts Institute of Technology Bates Linear Accelerator (BATES), the Saturne National Laboratory at Saclay (SATURNE), the Swiss Institue for Nuclear Research (SIN), and the TRIUMF Meson Reasearch Facility at Vancouver (TRIUMF). Roughly speaking, this body of data consists of In'lear scattering and reactions produced by beams of electrons, pions, nucleons, and light-ions with bombarding energies ranging from 100-1000 MeV. The fieid of medium-energy nuclear physics, which is essentially defined by this body of data, is recognized as a separate and distinct discipline, in spite of the obvious areas of overlap with low-energy and high-energy physics. Unlike the fields of low-energy and high-energy physics, however, there has been no centralized effort towards the compilation of medium-energy data.

To remedy this situation we propuse the formation of a Medium-Energy Nuclear Data LIBrary (MENDLIB). The proposed project would allow users to access a compilation of medium-energy experimental data and references via an on-line computer facility. The establishment of such a project would greatly reduce duplication of effort, help to guide future avenues of research, and ensure that the maximum benefit can be derived from the nation's investment in medium-energy physics.

\section{A. Benefits from Project}

A compilation of experimental data and references such as the one we are proposing would be of use to the entire medium-energy nuclear physics community - theorists, experimentalists, and applied researchers.

For a theorist to adequately evaluate his own calculations, he must have access to the most accurate and most recent experimental data. However, the volume of data is large, and the task of keeping up with the latest measurements is difficult. The availability of an experimental data service will eliminate the need for each theorist to be his own data manager. Furthermore, a computerized data library of the type we propose will facilitate the manipulation of large quantities of related data, so that systematic trends in the data can be more easily detected. The observation of systematic trends often leads to a better understanding of fundamental processes and, in turn, 
suggests new areas for investigation.

For an experimentalist to plan an experiment or an experimental program, he needs to know what measurements have been made and to what accuracy. This generally entails a time-consuming literiture search through the journals in which medium-energy data are published. Much of this effort could be eliminated if a central library of experimental data and references was available. Such a data service may also assist accelerator scheduling ccmmittees in assessing the relative merits of experimental proposals.

A medium-energy experimental data library would also be of assistance to the applied researcher because it would provide an orderly and complete collection of available data pertinent to a particular application. Examples of such data include:

- Neutral-particle cross sections for shielding calculations.

- Pion production cross sections (electron and proton induced) to determine optimum parameters for future accelerators and/or beam lines.

- Spallation reaction yields for isotope production and radiation damage studies using medium-energy bearns.

\section{B. Timeliness of Project}

The formation of a medium-energy experimental data library is long overdue. Not only will it ensure the broadest distribution of the last decade's worth of data, it will be ready to accept new data from the next generation of experiments. Given the trend towards limited future resources, an experimental data service such as the one we are proposing will undoubtedly influence the future of nuclear physics. The impact on the following future initiatives is worth considering (and perhaps expanding upon in a later draft):

- Data from IUCF's cooler and BATES upgrade,

- Connections with CEBAF and RHIC data, and

- LAMPF's upgrade to higher-energy pions.

\section{Objectives}

Simply stated, the goal of this project is to provide a comprehensive, on-line, computerized compilation of medium-energy experimental data and references. During the design and implementation, the primary consideration 
will be the user. This will ensure that experts and non-experts alike will have easy access to the data. In this section we outline the overall scope of the project, listing the data to be included and levels of computer $c^{\wedge \text { r }}$ development for accessing the data. We divide the project into three phases, each of limited scope and building upon one another.

\section{Phase I: the Pilot Project.}

- Data Included: selected pion and proton measured cross sections for elastic, charge-exchange (single and double), inelastic (to discrete and giant resonances), inclusive, and pion absorption and production.

- Code Development: a menu-driven program in a user-friendly environment with dial-up communication available to validated LAMPF computer users.

The goal of this initial phase of the MENDLIB project is to demonstrate the feasibility of the project using existing personnel, software, and computing equipment. The data to be included in this phase will be limited to a select amount of LAMPF data, and access will be limited to users of the LAMPF computers. The resources needed for this pilot phase will be supplied by the Medium-Energ. (MP) and the Theoretical (T) divisions of the Los Alamos National Laboratory. After this phase of the project, the methods for attaining the goals of the future phases can be refined and the resources needed to attain those goals can be more accurately assessed.

\section{Phase II.}

- Data Included: nucleon and pion LAMPF experimental data not compiled in PHASE I and nucleon and pion experimental data from other facilities such as IUCF and TRIUMF.

- Code Development: expand program-user options and allow access to the nuclear physics community.

In this phase, official participation in the project by additional facilities (besides LAMPF) commences. This involvement will require the establishment of a dedicated MENDLIB representative $[\approx 20 \%$ full time employee (fte)] 
from the in-house personnel at the participating facilities. Also, a standalone, dedicated Micro-VAX system $(\approx \$ 50 \mathrm{~K}$ including peripheral equipment) will be required to implement this phase and the following phases of development.

\section{Phase III.}

- Data Included: electron data from BATES, medium energy light-ion data from facilities such as IUCF, Jülich, and SATURNE, and possibly kaon and anti-nucleon data.

- Code Development: continue to expand and/or modify projram-user options depending on user feedback, and add communication via a high-speed network.

Depending on the success of Phase II, the project can be expanded to become a truly international project with MENDLIB representatives at all the medium-energy facilities.

\section{Procedure and Resources for Phase I}

In this section we propose a course of action that would attain the above stated goals of Phase I of the MENDLIB project. This pilot phase of the project will be implemented through the use of existing personnel, software, and equipment at Los Alamos and through the cooperation of the MP and $\mathrm{T}$ division's groups (MP-1 and T-2). As mentioned earlier, the data to be included in this pilot phase of the project will be limited to selected LAMPF data, and access to this data will be limited to validated LAMPF computer users. The time period for development and implementation will be approximately six months (beginning around $6 / 1 / 87$ ), followed by another six months of usage and feedback. After a one year period (around 6/1/88), the success of Phase I of the MENDLIB project will be assessed and the decision to proceed with the subsequent phases of this project will be made.

\section{- Personnel:}

The initial commitment required is two high-level fte's at a $50 \%$ time involvement for the first six months (June-December 1987). One person will be from MP-1 and the other from T-2. During the second six month period (January 1987-June 1988), the time involvement will of 
these two people will be reduced to a $20 \%$ level. Coordination of this project will be directed by the authors (also from T-2).

- Software:

The INGRES data base management system will be used (this is already available at LAMPF), and a menu-driver for this system will be written. A period of three months (June-August 1987) will be used for developing the pilot program menu-driver. Over the next three months (September-November 1987), a call for data will be made to the LAMPF users by means of the LAMPF Newsletter, and the pilot program will be tested. After this test period (around December 1, 1987), any validated user of the LAMPF computers will be allowed to execute the pilot program and access the available data.

- Equipment:

With the cooperation of MP-1, the existing VAX-cluster and dial-in modems at the data acquisition center of LAMPF will be used. We have estimated that the additional demands that would be placed on this sysiem by the pilot project (e.g., disk space, increase in telephone calls, increase in cpu) would not significantly interfere with normal operations.

In addition to the above, we feel that it is important to communicate our intentions to the nuclear physics community and to solirit comments and suggestions from this community. After all, the ultimate success of this project depends upon how well it will meet the needs of this community. Along these lines, we plan to: (1) solicit outside input by means of the various user group and American Physical Society Division of Nuclear Physics newsletters and (2) establish contacts at IUCF, TRIUMF, and BATES.

\section{Summary}

We have proposed the formation of an on-line computerized data library (MENDLIB) that would contain published medium-energy experimental data and that would serve the needs of the medium-energy nuclear physics community. We have described in detail a pilot project (called Phase Ij that would be an initial step towards attaining the goal of a comprehensive and user-friendly data library. The objective of Phase I is to demonstrate the 
feasibility of attaining the goals of a larger project by using existing personnel, software, and computing equipment to attain a more limited goal. The data to be included in this phase will be limited to a select amount of LAMPF data, and access will be limited to users of the LAMPF computers. The resources needed for this pilot phase will be supplied by the MediumEnergy and Theoretical divisions' groups, MP-1 and T-2, of the Los Alamos National Laboratory. The time period for development and implementation will be approximately six months (beginning around 6/1/87), followed by another six months of usage and feedback. After a one year period (around $6 / 1 / 88$ ), the success of Phase I of the MENDLIB project will be assessed and the decision to proceed with the subsequent phases of this project will be made. 